\title{
ON APPROXIMATE CONFORMAL MAPPING OF A DISK AND AN ANNULUS WITH RADIAL AND CIRCULAR SLITS ONTO MULTIPLY CONNECTED DOMAINS
}

\author{
Pyotr N. Ivanshin, Elena A. Shirokova \\ Kazan Federal University \\ Kazan, Russia \\ pivanshi@yandex.ru,Elena.Shirokova@kpfu.ru \\ Received: 29 March 2020; Accepted: 17 June 2020
}

\begin{abstract}
The method of boundary curve reparametrization is generalized to the case of multiply connected domains. We construct the approximate analytical conformal mapping of the unit disk with $m$ circular slits and $n-m$ radial slits and an annulus with $(m-1)$ circular slits and $n-m$ radial slits onto an arbitrary given $(n+1)$ multiply connected finite domain with a smooth boundary. The method is based on extension of the Lichtenstein-Gershgorin equation to a multiply connected domain. The proposed method is reduced to the solution of a linear system with unknown Fourier coefficients. The approximate mapping function has the form of a Cauchy integral. Numerical examples demonstrate that the proposed method is effective in computations.
\end{abstract}

MSC 2010: 30C20, 45B05

Keywords: conformal mapping, multiply connected domain, Fredholm integral equation

\section{Introduction}

Conformal mappings by the analytical functions of a complex variable play an important role in the solution of many problems of mechanics and mathematics, particulary in the case of plane potential fields and the Laplace equation solution [1]. The conformal mapping of the circular domain (a disk with circular and radial slits or an annulus with circular and radial slits) onto a multiply connected domain can be applied to the solution of plane boundary value problems for corresponding domains by the Poincare series $[2,3]$ or equivalently by the Schottky-Klein prime function [4]. The existence and uniqueness of the solution for similar problems under certain assumptions is a consequence of the results of [5]. Computer progress has stimulated the appearance of many numerical methods for conformal mapping constructions [6,7]. For example, the widely-used Wegmann numerical method is based on the Riemann-Hilbert problem solution and involves iteration processes $[8,9]$. Some authors searched for boundary reparametrization with linearization of this process as in $[10,11]$. Many authors apply the integral equations which 
contain the singular integrals. The collocation method or Nyström's method can be applied for the solution of such integral equations [12].

Several types of canonical regions exist for conformal mappings [12]. Nasser managed to map bounded and unbounded multiply connected regions onto these five canonical regions by reformulating the mapping function as a Riemann-Hilbert problem which is solved by means of a boundary integral equation with the generalized Neumann kernel [12]. The right-hand side of the integral equation involves the integral with cotangent singularity which is approximated by Wittich's method. The integral equation was discretized by the Nyström method with the trapezoidal rule to obtain a linear system [12-18].

Here we continue the constructions of [19] and present a new method of the approximate conformal mapping of the unit disk with circular and radial slits and an annulus with circular and radial slits onto a multiply connected domain with a smooth boundary. We apply a method of integral equations generalising the Lichtenstein-Gershgorin one with a Neumann kernel obtained from the necessary and sufficient condition for a function defined at the points of a smooth contour to be the boundary values of some function analytical in the correspondent domain. These equations were thoroughly described in $[7,20]$. We give the approximate solution of these equations by reduction to a linear system as in $[13,14,19]$, therefore the method is easily programmable.

\section{Approximate conformal mapping of the circular domains of two types onto a multiply connected domain by means of boundary reparametrization}

Consider a finite $(n+1)$ - connected domain $D_{z}$ bounded by the outer simple smooth curve $L_{0}$ and the inner simple smooth curves $L_{s}$ given by the equations

$$
L_{s}=\left\{z=z_{s}(t), z_{s}(0)=z_{s}(2 \pi), t \in[0,2 \pi]\right\}, s=0, \ldots, N .
$$

We also assume that the boundary curves $L_{s}$ complex representations are as follows:

$$
z_{s}(t)=\sum_{k=-m_{s}}^{n_{s}} d_{k s} e^{i k t}, t \in[0,2 \pi], s=0, \ldots, N .
$$

The parametrization traces the domain $D_{z}$ along $L_{0}$ counterclockwise and along the inner contours $L_{s}, s=1, \ldots, n$, clockwise.

Definition. We call the unit disk with $m$ circular slits $\zeta=R_{s} e^{i \theta}, \theta_{1 s}<\theta<\theta_{2 s}$, $0<R_{s}<1, \theta_{2 s}-\theta_{1 s}<2 \pi, s=1, \ldots, m$, and with $n-m$ radial slits $\zeta=\operatorname{Re}^{i \theta_{j}}, 0<$ $R_{1 j}<R<R_{2 j}<1,0<\theta_{j}<2 \pi, j=m+1, \ldots, n$, an $(n+1)$ - connected canonical domain of the first type. We call the annulus with the exterior radius 1 , with the interior radius $r, r<1$, and with $(m-1)$ circular slits $\zeta=R_{s} e^{i \theta}, \theta_{1 s}<\theta<\theta_{2 s}$, 
$r<R_{s}<1, \theta_{2 s}-\theta_{1 s}<2 \pi, s=1, \ldots, m-1$, and with $n-m$ radial slits $\zeta=R e^{i \theta_{j}}, 0<$ $R_{1 j}<R<R_{2 j}<1,0<\theta_{j}<2 \pi, j=m+1, \ldots, n$, an $(n+1)$ - connected canonical domain of the second type.

Theorem 1 Analytic-numerical approximate conformal mapping exists for an $(n+1)$-connected circular domain $D_{\zeta}$ of the first type such that the function $f(\zeta)$ maps conformally the domain $D_{\zeta}$ onto the given $(n+1)$-connected domain $D_{z}$ with smooth boundary components. The approximate solution converges to the exact one as $O\left(1 / N^{2}\right)$. Here $N$ is the size of the truncated auxiliary matrix.

PROOF Existence of solution is a known fact [5], so we concentrate on the approximate solution construction and proof of its convergence. The map is unique under the following conditions: $f(0)=A+i B, f(1)=C+i D,(A, B) \in D_{z},(C, D) \in L_{0}$.

We assume that $0 \in D_{z}$ and $A+i B=0$ without loss of generality. We give the constructive proof. We construct the conformal map of the circular domain of the first type onto the domain $D_{z}$ by reparametrization of the given boundary representations. So we search for the function $t_{0}(\theta), \theta \in[0,2 \pi]$, for the functions $t_{s}(\theta), s=1, \ldots, m$, $\theta \in\left[\theta_{1 s}, \theta_{2 s}\right]$, and for the functions $t_{j}(R), R \in\left[R_{1 j}, R_{2 j}\right]$, such that the values $z_{s}\left(t_{s}(\theta)\right)$, $s=0, \ldots, m, z_{j}\left(t_{j}(R)\right)$, be the boundary values of an analytic function in the corresponding circular domain. The parameters $R_{s}, \theta_{1 s}, \theta_{2 s}, s=1, \ldots, m, R_{1 j}, R_{2 j}, \theta_{j}$, $j=m+1, \ldots, n$, are also unknown and will be found within the solution process.

Let us consider the analytic in the domain $D_{z}$ function $\zeta(z)$ which maps conformally the domain $D_{z}$ onto $D_{\zeta}$ with the correspondence $\zeta(0)=0$ and the analytic in $D_{z}$ function $\log \frac{z}{\zeta}$. According to [21], the necessary and sufficient condition for $\log \frac{z}{\zeta}$ to be analytic in $D_{z}$ are the boundary relations

$$
\begin{gathered}
\log \frac{z_{s}(t)}{R_{s} e^{i \theta_{s}(t)}}=\sum_{\sigma=0}^{m} \frac{1}{\pi i} \int_{0}^{2 \pi} \log \frac{z_{\sigma}(\tau)}{R_{\sigma} e^{i \theta_{\sigma}(\tau)}}\left[\log \left(z_{\sigma}(\tau)-z_{s}(t)\right)\right]_{\tau}^{\prime} d \tau+, \\
\sum_{j=m+1}^{n} \frac{1}{\pi i} \int_{0}^{2 \pi} \log \frac{z_{j}(\tau)}{R_{j}(\tau) e^{i \theta_{j}}}\left[\log \left(z_{j}(\tau)-z_{s}(t)\right)\right]_{\tau}^{\prime} d \tau,
\end{gathered}
$$

where $t \in[0,2 \pi], s=0, \ldots, m, R_{0}=1$, and

$$
\begin{gathered}
\log \frac{z_{j}(t)}{R_{j}(t) e^{i \theta_{j}}}=\sum_{s=0}^{m} \frac{1}{\pi i} \int_{0}^{2 \pi} \log \frac{z_{s}(\tau)}{R_{s} e^{i \theta_{s}(\tau)}}\left[\log \left(z_{s}(\tau)-z_{j}(t)\right)\right]_{\tau}^{\prime} d \tau+, \\
\sum_{k=m+1}^{n} \frac{1}{\pi i} \int_{0}^{2 \pi} \log \frac{z_{k}(\tau)}{R_{k}(\tau) e^{i \theta_{k}}}\left[\log \left(z_{k}(\tau)-z_{j}(t)\right)\right]_{\tau}^{\prime} d \tau,
\end{gathered}
$$


where $t \in[0,2 \pi], j=m+1, \ldots, n$.

We introduce the following functions: $q_{s}(t)=\arg z_{s}(t)-\theta_{s}(t)$, where $\theta_{s}(t)$ is the polar angle of the image of the point of $z_{s}(t), s=0, \ldots, m$, and $p_{j}(t)=\log \left|z_{j}(t)\right|-$ $\log R_{j}(t)$, where $R_{j}(t)$ is the radius of the image of the point of $z_{j}(t), j=m+1, \ldots, n$.

We separate the imaginary part of both sides of equation (1) and arrive to the equation system described in [3]:

$$
\begin{aligned}
q_{s}(t)=\sum_{\sigma=0}^{m} \frac{1}{\pi} \int_{0}^{2 \pi} q_{\sigma}(\tau)\left[\arg \left(z_{\sigma}(\tau)-z_{s}(t)\right)\right]_{\tau}^{\prime} d \tau-\sum_{\sigma=0}^{m} \frac{1}{\pi} \int_{0}^{2 \pi} \log \frac{\left|z_{\sigma}(\tau)\right|}{R_{\sigma}}\left[\log \left|z_{\sigma}(\tau)-z_{s}(t)\right|\right]_{\tau}^{\prime} d \tau+ \\
\quad+\sum_{j=m+1}^{n} \frac{1}{\pi} \int_{0}^{2 \pi}\left[\arg z_{j}(\tau)-\theta_{j}\right]\left[\arg \left(z_{j}(\tau)-z_{s}(t)\right)\right]_{\tau}^{\prime} d \tau- \\
-\sum_{j=m+1}^{n} \frac{1}{\pi} \int_{0}^{2 \pi} p_{j}(\tau)\left[\log \left|z_{j}(\tau)-z_{s}(t)\right|\right]_{\tau}^{\prime} d \tau, \quad s=0, \ldots, m .
\end{aligned}
$$

This system generalizes the Lichtenstein-Gershgorin equation for non simple-connected regions.

We separate the real part of both sides of equation (2):

$$
\begin{gathered}
p_{j}(t)=\sum_{s=0}^{m} \frac{1}{\pi} \int_{0}^{2 \pi}\left[\log \left|z_{s}(\tau)\right|-\log R_{s}\right]\left[\arg \left(z_{s}(\tau)-z_{j}(t)\right)\right]_{\tau}^{\prime} d \tau+ \\
+\sum_{s=0}^{m} \frac{1}{\pi} \int_{0}^{2 \pi} q_{s}(\tau)\left[\log \left|z_{s}(\tau)-z_{j}(t)\right|\right]_{\tau}^{\prime} d \tau+\sum_{k=m+1}^{n} \frac{1}{\pi} \int_{0}^{2 \pi} p_{k}(\tau)\left[\arg \left(z_{k}(\tau)-z_{j}(t)\right)\right]_{\tau}^{\prime} d \tau+ \\
+\sum_{k=m+1}^{n} \frac{1}{\pi} \int_{0}^{2 \pi}\left[\arg \left[z_{k}(\tau)-\theta_{k}\right]\left[\log \left|z_{k}(\tau)-z_{j}(t)\right|\right]_{\tau}^{\prime} d \tau, \quad j=m+1, \ldots, n .\right.
\end{gathered}
$$

After differentiating relations (3) and (4) with respect to $t$ and integrating the results by parts, we obtain the following relations on the functions $q_{s}^{\prime}(t)$ and $p_{j}^{\prime}(t)$ respectively:

$$
q_{s}^{\prime}(t)=\sum_{\sigma=0}^{m} \frac{1}{\pi} \int_{0}^{2 \pi} q_{\sigma}^{\prime}(\tau) K_{\sigma, s}(\tau, t) d \tau+\sum_{j=m+1}^{n} \frac{1}{\pi} \int_{0}^{2 \pi} p_{j}^{\prime}(\tau) L_{j, s}(\tau, t) d \tau+Q_{s}(t), s=0, \ldots, m,
$$


$p_{j}^{\prime}(t)=-\sum_{s=0}^{m} \frac{1}{\pi} \int_{0}^{2 \pi} q_{s}^{\prime}(\tau) L_{s, j}(\tau, t) d \tau+\sum_{k=m+1}^{n} \frac{1}{\pi} \int_{0}^{2 \pi} p_{k}^{\prime}(\tau) K_{k, j}(\tau, t) d \tau+P_{j}(t), j=m+1, \ldots, n$,

where

$$
\begin{gathered}
K_{\sigma s}(\tau, t)=-\left[\arg \left(z_{\sigma}(\tau)-z_{s}(t)\right)\right]_{t}^{\prime}, \quad L_{j, s}(\tau, t)=\left[\log \left(z_{j}(\tau)-z_{s}(t)\right)\right]_{t}^{\prime}, \\
Q_{s}(t)=\sum_{\sigma=0}^{m} \frac{1}{\pi} \int_{0}^{2 \pi}\left[\log \left|z_{\sigma}(\tau)\right|\right]^{\prime} L_{\sigma, s}(\tau, t) d \tau+\sum_{j=m+1}^{n} \frac{1}{\pi} \int_{0}^{2 \pi}\left(\arg z_{j}(\tau)\right)^{\prime} K_{j, s}(\tau, t) d \tau, \\
P_{j}(t)=\sum_{s=0}^{m} \frac{1}{\pi} \int_{0}^{2 \pi}\left[\log \left|z_{s}(\tau)\right|\right]^{\prime} K_{s, j}(\tau, t) d \tau-\sum_{k=m+1}^{n} \frac{1}{\pi} \int_{0}^{2 \pi}\left(\arg z_{k}(\tau)\right)^{\prime} L_{k, j}(\tau, t) d \tau .
\end{gathered}
$$

The kernel $L_{\sigma, s}$ has a singularity in the form of $\cot \frac{\tau-t}{2}$ for $\sigma=s$ :

$$
\begin{gathered}
\left(\log \left|z_{s}(\tau)-z_{s}(t)\right|\right)_{t}^{\prime}=\Re\left(\log \sum_{k=-m_{s}}^{n_{s}} d_{k s}\left[e^{i k \tau}-e^{i k t}\right]\right)_{t}^{\prime}=\Re\left(\log \sin \frac{\tau-t}{2}+\right. \\
\left.+\log \left[\sum_{k=1}^{n_{s}} d_{k s} e^{i k t} \sum_{l=0}^{k-1} e^{i l(\tau-t)}-\sum_{k=1}^{m_{s}} d_{(-k) s} e^{-i k \tau} \sum_{l=0}^{k-1} e^{i l(\tau-t)}\right]\right)_{t}^{\prime}= \\
=-\frac{1}{2} \cot \frac{\tau-t}{2}+\left(\log \left|\sum_{k=1}^{n_{s}} d_{k s} e^{i k t} \sum_{l=0}^{k-1} e^{i l(\tau-t)}-\sum_{k=1}^{m_{s}} d_{(-k) s} e^{-i k \tau} \sum_{l=0}^{k-1} e^{i l(\tau-t)}\right|\right)_{t}^{\prime}
\end{gathered}
$$

The Cauchy principal value integral $\frac{1}{\pi} \int_{0}^{2 \pi}\left[\log \left|z_{\sigma}(\tau)\right|\right]^{\prime} \cot \frac{\tau-t}{2} d \tau$ can be calculated via Hilbert formula [15] as in [14]. Finally, we obtain the following system of Fredholm integral equations of the second kind which can be written in the operator form as follows:

$$
\left(\begin{array}{ccccccc}
I-K_{0,0} & -K_{1,0} & \ldots & -K_{m, 0} & -L_{m+1,0} & \ldots & -L_{n, 0} \\
-K_{0,1} & I-K_{1,1} & \ldots & -K_{m, 1} & -L_{m+1,1} & \ldots & -L_{n, 1} \\
\ldots & \ldots & \ldots & \ldots & \ldots & \ldots & \ldots \\
-K_{0, m} & -K_{1, m} & \ldots & I-K_{m, m} & -L_{m+1, m} & \ldots & -L_{n, m} \\
L_{0, m+1} & L_{1, m+1} & \ldots & L_{m, m+1} & I-K_{m+1, m+1} & \ldots & -K_{n, m+1} \\
\ldots & \ldots & \ldots & \ldots & \ldots & \ldots & \ldots \\
L_{0, n} & L_{1, n} & \ldots & L_{m, n} & -K_{m+1, n} & \ldots & I-K_{n, n}
\end{array}\right)\left(\begin{array}{c}
q_{0}^{\prime} \\
\ldots \\
q_{m}^{\prime} \\
p_{m+1}^{\prime} \\
\ldots \\
p_{n}^{\prime}
\end{array}\right)=
$$




$$
=\left(Q_{0}, \ldots, Q_{m}, P_{m+1}, \ldots, P_{n}\right)^{T}
$$

The last operator system can be reduced to the infinite linear system over the Fourier coefficients of the unknown functions $q_{s}^{\prime}(t), s=0, \ldots, m, p_{j}^{\prime}(t), j=m+$ $+1, \ldots, n$, if we find the coefficients of double Fourier expansions of the kernels of integral operators and compare the coefficients with the same trigonometric functions [13]. The approximate solution of the infinite system over Fourier coefficients of the unknown functions is a solution of a truncated system over the Fourier coefficients of the unknown functions.

Convergence of the approximate solution of system (5)-(6) to the exact one provided $M \rightarrow \infty$ was proved in [13] for the case of conformal mapping of a simply connected domain. This proof can be applied to the case of multiply connected domain if we replace the corresponding space $l^{2}$ by the space $l^{2} \times l^{2} \times \ldots \times l^{2}$.

We search for the approximate solution of system (5)-(6) in the form of Fourier polynomials:

$$
q_{s}^{\prime}(t)=\sum_{l=1}^{M} \alpha_{l s} \cos l t+\beta_{l s} \sin l t, s=0, \ldots, m, p_{j}^{\prime}(t)=\sum_{l=1}^{M} \alpha_{l j} \cos l t+\beta_{l j} \sin l t
$$

Here $j=m+1, \ldots, n, t \in[0,2 \pi]$.

Now integral Fredholm equations of the second kind in (5) and (6) can be reduced to the linear system over Fourier coefficients $\alpha_{l s}$ and $\beta_{l s}, s=0, \ldots, m, \alpha_{l j}$ and $\beta_{l j}$, $j=m+1, \ldots, n$ :

$$
\begin{aligned}
\left(\begin{array}{ccccccccc}
A_{00} & B_{00} & A_{01} & \ldots & B_{0 n} & E_{0 m+1} & F_{0 m+1} & \ldots & E_{0 n} \\
C_{00} & D_{00} & C_{01} & \ldots & D_{0 m} & G_{0 m+1} & H_{0 m+1} & \ldots & H_{0 n} \\
\vdots & \vdots & \vdots & \ddots & \vdots & \vdots & \ddots & \vdots & \\
C_{m 0} & D_{m 0} & C_{m 1} & \ldots & D_{m m} & G_{m m+1} & H_{m m+1} & \ldots & H_{m n} \\
P_{m+10} & Q_{m+10} & P_{m+11} & \ldots & Q_{m+1 m} & R_{m+1 m+1} & S_{m+1 m+1} & \ldots & S_{m+1 n} \\
N_{m+10} & T_{m+10} & N_{m+11} & \ldots & T_{m+1 m} & V_{m+1 m+1} & U_{m+1 m+1} & \ldots & U_{m+1 n} \\
\vdots & \vdots & \vdots & \ddots & \vdots & \vdots & \ddots & \vdots & \\
N_{n 0} & T_{n 0} & N_{n 1} & \ldots & T_{n m} & V_{n m+1} & U_{n m+1} & \ldots & U_{n n}
\end{array}\right) \times\left(\begin{array}{c}
\alpha_{0} \\
\beta_{0} \\
\alpha_{1} \\
\beta_{1} \\
\vdots \\
\beta_{n}
\end{array}\right)= \\
\\
\end{aligned}
$$

where $\alpha_{s}=\left(\alpha_{1 s}, \ldots, \alpha_{n s}\right)^{T}, \beta_{s}=\left(\beta_{1 s}, \ldots, \beta_{n s}\right)^{T}$. The vectors $a_{s}=\left(a_{1 s}, \ldots, a_{n s}\right)^{T}$, $b_{s}=\left(b_{1 s}, \ldots, b_{n s}\right)^{T}$ on the right-hand side of the system consist of the elements

$$
a_{j s}=\frac{1}{\pi} \int_{0}^{2 \pi} Q_{s}(t) \cos j t d t, b_{j s}=\frac{1}{\pi} \int_{0}^{2 \pi} Q_{s}(t) \sin j t d t, j=1, \ldots, m, s=0, \ldots, m,
$$


$a_{j k}=\frac{1}{\pi} \int_{0}^{2 \pi} P_{k}(t) \cos j t d t, b_{j k}=\frac{1}{\pi} \int_{0}^{2 \pi} P_{k}(t) \sin j t d t, j=1, \ldots, m, k=m+1, \ldots, n$,

The block matrices $A_{\sigma s}, B_{\sigma s}, C_{\sigma s}, D_{\sigma s}, E_{\sigma l}, F_{\sigma l}, G_{\sigma l}, H_{\sigma l}, P_{p s}, Q_{p s}, N_{p s}, T_{p s}, R_{p l}$, $S_{p l}, V_{p l}, U_{p l}, \sigma, s=0, \ldots, m, l, p=m+1, \ldots, n$ of size $M \times M$ consist of the elements

$A_{\sigma s j k}=\delta_{\sigma s} \delta_{j k}-\frac{1}{\pi^{2}} \int_{0}^{2 \pi} \cos k \tau d \tau \int_{0}^{2 \pi} K_{\sigma s}(\tau, t) \cos j t d t, B_{\sigma s j k}=-\frac{1}{\pi^{2}} \int_{0}^{2 \pi} \sin k \tau d \tau \int_{0}^{2 \pi} K_{\sigma s}(\tau, t) \cos j t d t$,

$C_{\sigma s j k}=-\frac{1}{\pi^{2}} \int_{0}^{2 \pi} \cos k \tau d \tau \int_{0}^{2 \pi} K_{\sigma s}(\tau, t) \sin j t d t, D_{\sigma s j k}=\delta_{\sigma s} \delta_{j k}-\frac{1}{\pi^{2}} \int_{0}^{2 \pi} \sin k \tau d \tau \int_{0}^{2 \pi} K_{\sigma s}(\tau, t) \sin j t d t$

$E_{\sigma l j k}=-\frac{1}{\pi^{2}} \int_{0}^{2 \pi} \cos k \tau d \tau \int_{0}^{2 \pi} L_{\sigma l}(\tau, t) \cos j t d t, F_{\sigma l j k}=-\frac{1}{\pi^{2}} \int_{0}^{2 \pi} \sin k \tau d \tau \int_{0}^{2 \pi} L_{\sigma l}(\tau, t) \cos j t d t$,

$G_{\sigma l j k}=-\frac{1}{\pi^{2}} \int_{0}^{2 \pi} \cos k \tau d \tau \int_{0}^{2 \pi} L_{\sigma l}(\tau, t) \sin j t d t, U_{p l j k}=\delta_{p l} \delta_{j k}-\frac{1}{\pi^{2}} \int_{0}^{2 \pi} \sin k \tau d \tau \int_{0}^{2 \pi} K_{p l}(\tau, t) \sin j t d t$,

$H_{\sigma l j k}=-\frac{1}{\pi^{2}} \int_{0}^{2 \pi} \sin k \tau d \tau \int_{0}^{2 \pi} L_{\sigma l}(\tau, t) \sin j t d t, P_{p s j k}=\frac{1}{\pi^{2}} \int_{0}^{2 \pi} \cos k \tau d \tau \int_{0}^{2 \pi} L_{p s}(\tau, t) \cos j t d t$,

$Q_{p s j k}=\frac{1}{\pi^{2}} \int_{0}^{2 \pi} \sin k \tau d \tau \int_{0}^{2 \pi} L_{p s}(\tau, t) \cos j t d t, N_{p s j k}=\frac{1}{\pi^{2}} \int_{0}^{2 \pi} \cos k \tau d \tau \int_{0}^{2 \pi} L_{p s}(\tau, t) \sin j t d t$,

$T_{p s j k}=\frac{1}{\pi^{2}} \int_{0}^{2 \pi} \sin k \tau d \tau \int_{0}^{2 \pi} L_{p s}(\tau, t) \sin j t d t, R_{p l j k}=\delta_{p l} \delta_{j k}-\frac{1}{\pi^{2}} \int_{0}^{2 \pi} \cos k \tau d \tau \int_{0}^{2 \pi} K_{p l}(\tau, t) \cos j t d t$,

$S_{p l j k}=-\frac{1}{\pi^{2}} \int_{0}^{2 \pi} \sin k \tau d \tau \int_{0}^{2 \pi} K_{p l}(\tau, t) \cos j t d t, V_{p l j k}=-\frac{1}{\pi^{2}} \int_{0}^{2 \pi} \cos k \tau d \tau \int_{0}^{2 \pi} K_{p l}(\tau, t) \sin j t d t$,

where $j, k=1, \ldots, m, \delta_{r t}$ is the Kronecker delta function. 
The functions $q_{s}(t), s=0, \ldots, m$, and $p_{j}(t), j=m+1, \ldots, n$, can be restored via their derivatives (7) with an arbitrary constant summand

$$
\begin{gathered}
q_{s}(t)=q_{0 s}+\tilde{q}_{s}(t), \quad \tilde{q}_{s}(t)=\sum_{l=1}^{M} \frac{\alpha_{l s}}{l} \sin l t-\frac{\beta_{l s}}{l} \cos l t \\
p_{j}(t)=p_{0 j}+\tilde{p}_{j}(t), \quad \tilde{p}_{j}(t)=\sum_{l=1}^{M} \frac{\alpha_{l j}}{l} \sin l t-\frac{\beta_{l s}}{l} \cos l t, \quad t \in[0,2 \pi] .
\end{gathered}
$$

We choose the constant summand $q_{00}$ in accordance with the condition $f(1)=C+i D$ in the following way. We find the value of the parameter $\hat{t}$ such that $z_{0}(\hat{t})=C+i D$. Now $q_{00}=\arg (C+i D)-\tilde{q}_{0}(\hat{t})$.

We obtain the values of the other constant summands $q_{0 s}, s=1, \ldots, m, p_{0 j}, j=$ $=m+1, \ldots, n$, and also the values of $R_{s}, s=1, \ldots, m, \theta_{j}, j=m+1, \ldots, n$, in the following way. We take $n$ points in each of $n$ finite component of the set complement of $D_{z}$. Let us denote these points $z_{k}^{*}, k=1, \ldots, n$. The function $\log \frac{z_{s}(t)}{R_{s} e^{i \theta(t)}}$, $s=0, \ldots, m, \log \frac{z_{j}(t)}{R(t) e^{i \theta_{j}(t)}}, j=m+1, \ldots, n$, is the boundary value of the analytical in $D_{z}$ function, so the Cauchy integral with the corresponding density along the boundary of $D_{z}$ vanishes at the points $z_{j}^{*}, j=1, \ldots, n$. Therefore, we have the linear complex system

$$
\begin{gathered}
\sum_{\sigma=0}^{m} \frac{1}{2 \pi i} \int_{0}^{2 \pi}\left[i q_{0 \sigma}-\log R_{\sigma}+\log \left|z_{\sigma}(\tau)\right|+i \tilde{q}_{s}(\tau)\right]\left[\log \left(z_{\sigma}(\tau)-z_{j}^{*}\right)\right]_{\tau}^{\prime} d \tau+ \\
\sum_{k=m+1}^{n} \frac{1}{2 \pi i} \int_{0}^{2 \pi}\left[-i \theta_{k}+i \arg z_{k}(\tau)+p_{0 k}+\tilde{p}_{k}(\tau)\right]\left[\log \left(z_{k}(\tau)-z_{j}^{*}\right)\right]_{\tau}^{\prime} d \tau=0, j=1, \ldots, n,
\end{gathered}
$$

with the unknown real $q_{0 \sigma}, \log R_{\sigma}, \sigma=1, \ldots, m, p_{0 k}, \theta_{k}, k=m+1, \ldots, n$.

We restore the values of $\theta_{1 j}, \theta_{2 j}, j=1, \ldots, m$, after we have restored $q_{0 j}$. Then

$$
\theta_{1 j}=\min _{t \in[0,2 \pi]}\left[\arg z_{i}(t)-\tilde{q}_{j}(t)\right]-q_{0 j}, \theta_{2 j}=\max _{t \in[0,2 \pi]}\left[\arg z_{i}(t)-\tilde{q}_{j}(t)\right]-q_{0 j} .
$$

We restore the values of $R_{1 j}, R_{2 j}, j=m+1, \ldots, n$, after we have restored $p_{0 j}$. Then $\log R_{1 j}=\min _{t \in[0,2 \pi]}\left[\log \left|z_{j}(t)\right|-\tilde{p}_{j}(t)\right]-p_{0 j}, \log R_{2 j}=\max _{t \in[0,2 \pi]}\left[\log \left|z_{j}(t)\right|-\tilde{p}_{j}(t)\right]-p_{0 j}$.

So all parameters of the circular domain of the first type $D_{\zeta}$ have been found.

Now we have the functions $q_{s}(t), s=0, \ldots, m, p_{k}(t), k=m+1, \ldots, n, t \in[0,2 \pi]$, and therefore we can restore the relations between the boundary parameters of the domains $D_{z}$ and $D_{\zeta}$ via the formulas $\theta_{s}(t)=\arg z_{s}(t)-q_{s}(t), s=0, \ldots, m, \log R_{k}(t)=$ 
$=\log \left|z_{k}(t)\right|-p_{k}(t), k=m+1, \ldots, n$. Note that $\theta_{0}(t)$ grows monotonically when $t$ grows from 0 to $2 \pi, \theta_{0}(2 \pi)-\theta_{0}(0)=2 \pi$, while each of the functions $\theta_{s}(t), s=$ $=1, \ldots, m, \log R_{k}(t), k=m+1, \ldots, n$, is $2 \pi$-periodic with one interval of increase and one interval of decrease. We can restore the inverse to $\theta_{0}(t)$ monotonically increasing function $t_{0}(\theta)$ and we can restore the single-valued functions $t_{s}^{ \pm}(\theta)$, $\theta \in\left[\theta_{1 s}, \theta_{2 s}\right], s=1, \ldots, m$, and $t_{s}^{ \pm}(R), R \in\left[R_{1 k}, R_{2 k}\right], k=m+1, \ldots, n$.

The approximate analytical function which maps $D_{\zeta}$ onto $D_{z}$ now has the form of the Cauchy integral

$$
\begin{gathered}
f(\zeta)=\frac{1}{2 \pi} \int_{0}^{2 \pi} \frac{z_{0}\left(t_{0}(\theta)\right) e^{i \theta} d \theta}{e^{i \theta}-\zeta}+\sum_{s=1}^{m} \frac{1}{2 \pi} \int_{\theta_{1 s}}^{\theta_{2 s}} \frac{\left.z_{s}\left(t_{s}^{+}(\theta)\right)-z_{s}\left(t_{s}^{-}(\theta)\right)\right] R_{s} e^{i \theta}}{R_{s} e^{i \theta}-\zeta} d \theta+ \\
+\sum_{j=m+1}^{n} \frac{1}{2 \pi i} \int_{R_{1 j}}^{R_{2 s}} \frac{\left[z_{j}\left(t_{j}^{+}(R)\right)-z_{j}\left(t_{j}^{-}(R)\right)\right] e^{i \theta_{j}}}{\operatorname{Re}^{i \theta_{j}}-\zeta} d R .
\end{gathered}
$$

We can apply the Cauchy integral in the form

$$
\begin{gathered}
f(\zeta)=\frac{1}{2 \pi} \int_{0}^{2 \pi} \frac{z_{0}(t) e^{i \theta_{0}(t)} \theta_{0}^{\prime}(t) d t}{e^{i \theta_{0}(t)}-\zeta}+\sum_{s=1}^{m} \frac{1}{2 \pi} \int_{0}^{2 \pi} \frac{z_{s}(t) R_{s} e^{i \theta_{s}(t)} \theta_{s}^{\prime}(t)}{R_{s} e^{i \theta_{s}(t)}-\zeta} d t+ \\
+\sum_{k=m+1}^{n} \frac{1}{2 \pi i} \int_{0}^{2 \pi} \frac{z_{j}(t) R_{j}^{\prime}(t) e^{i \theta_{j}}}{R_{j}(t) e^{i \theta_{j}}-\zeta} d t .
\end{gathered}
$$

in order not to deal with the functions $t_{s}^{ \pm}(\theta)$ or $t_{j}^{ \pm}(R)$ and not to integrate along the different borders of the same slit.

The values of $f(\zeta)$ at the points of $D_{\zeta}$ close to the boundary can be calculated with the help of analytic continuation of the Cauchy integral as in [22].

Theorem 2 Analytic-numerical approximate conformal mapping exists for an $(n+1)$-connected circular domain $D_{\zeta}$ of the second type such that the function $f(\zeta)$ maps conformally the domain $D_{\zeta}$ onto the given $(n+1)$-connected domain $D_{z}$ with smooth boundary components. The approximate solution converges to the exact one as $O\left(1 / N^{2}\right)$. Here $N$ is the size of the truncated auxiliary matrix.

Proof Existence of the exact solution is a generalisation of the Riemann Theorem [5]. The map is unique under the following conditions: the image of the inner circle $|\zeta|=r$ is the boundary component $L_{j}, j \in\{1,2, \ldots, m\}, f(1)=C+i D,(C, D) \in L_{0}$.

We assume that $j=1$ and $\int_{0}^{2 \pi}\left(\arg z_{1}(t)\right)^{\prime} d t=-2 \pi$ without loss of generality. We construct the conformal map of the circular domain of the first type onto the domain 
$D_{z}$ by reparametrization of the given boundary representations. So, we search for functions $t_{s}(\theta), \theta \in[0,2 \pi], s=0,1$, for the functions, for the functions $t_{s}(\theta), \theta \in$ $\left[\theta_{1 s}, \theta_{2 s}\right], s=2, \ldots, m$, and for the functions $t_{j}(R), R \in\left[R_{1 j}, R_{2 j}\right], j=m+1, \ldots, n$. The construction is as the one for mapping of the circular domain of the first type.

We consider the analytic in the domain $D_{z}$ function $\zeta(z)$ which maps conformly the domain $D_{z}$ onto $D_{\zeta}$ and the analytic in $D_{z}$ function $\log \frac{z}{\zeta}$. We apply the necessary and sufficient conditions for $\log \frac{z}{\zeta}$ to be analytic in $D_{z}$ which are boundary relations (1) and (2) as above. We introduce the functions $q_{s}(t)=\arg z_{s}(t)-\theta_{s}(t), s=0, \ldots, m$ and $p_{j}(t)=\log \left|z_{j}(t)\right|-\log R_{j}(t), j=m+1, \ldots, n$. After separation of the imaginary or real parts of both sides of these equations, differentiation with respect to $\tau$ and integration by parts we have equations (3) and (4). We reduce the solution of the integral equations to the solution of a linear system with truncated matrices if we consider $q_{s}^{\prime}(t)$ representations (7). Now we restore the functions $q_{s}(t), p_{j}(t)$ as in (8). The constant summand $q_{00}$ can be restored in the same way as for the previous case. The values of $q_{0 s}, p_{0 j}$, and $R_{s}, \theta_{j}, s=1, \ldots, m, j=m+1, \ldots, n$, can also be restored as above with the help of the additional points $z_{s}^{*}, s=1, \ldots, n$, located in the exterior of the domain $D_{z}$. Note that $z_{1}^{*}=0$.

\section{Examples}

1. Elliptic domain with two elliptic holes. Consider the elliptic domain bounded by the curve $6 e^{i t}-e^{-i t}$ with the holes bounded by the curves $0.2 e^{i t}-0.8 e^{-i t}-3 i$ and $0.8 e^{i(-t+\pi / 4)}+0.2 e^{-i(-t-\pi / 4)}+3 i, t \in[0,2 \pi]$. We constructed the conformal mapping from the unit disk with two radial slits: over the interval $\left[0.25 e^{-i 1.578}, 0.82 e^{-i 1.578}\right]$ and over the interval $\left[0.27 e^{i 1.588}, 0.8 e^{i 1.588}\right]$, the former is the preimage of the lower ellipse and the latter is mapped into the upper one. We give the preimage of the domain, a part of the polar coordinate net and the solution result (Fig. 2).
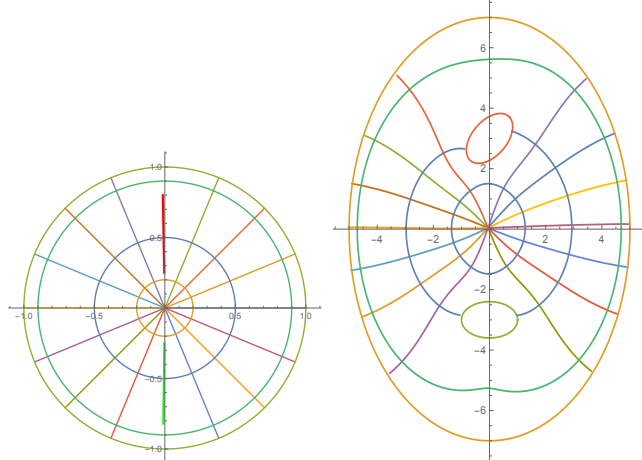

Fig. 1. The elliptic domain with two holes and radial slits 
2. Elliptic domain with two elliptic holes. Consider the elliptic domain bounded by the curve $6 e^{i t}-e^{-i t}$ with the holes bounded by the curves $0.2 e^{i t}-0.8 e^{-i t}-3 i$ and $0.8 e^{i(-t+\pi / 4)}+0.2 e^{-i(-t-\pi / 4)}+3 i, t \in[0,2 \pi]$. We constructed the conformal mapping from the unit disk with one radial and one circular slit: over the interval $\left[e^{0.93 i}, e^{2.18 i}\right]$ and over the interval $\left[0.27 e^{-i 1.588}, 0.84 e^{-i 1.588}\right]$, the former is the preimage of the lower ellipse and the latter is mapped into the upper one. We give the preimage of the domain, a part of the polar coordinate net and the solution result (Fig. 2).
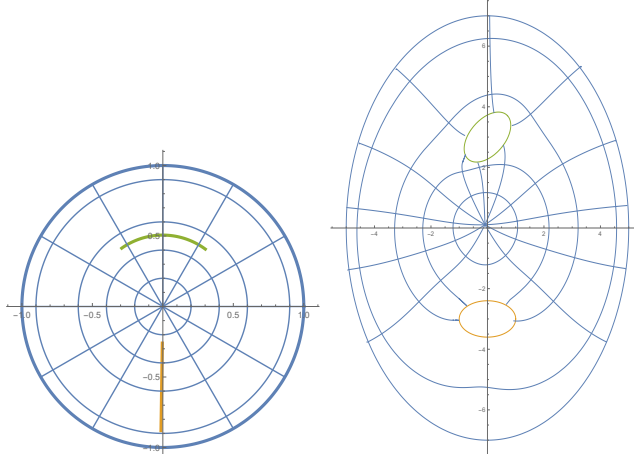

Fig. 2. The elliptic domain with two holes; circular and radial slits

\section{Conclusions}

Our method of the approximate solution construction is based on linear integral equations. These equations are reduced to an infinite linear system over Fourier coefficients of unknown conjugate functions. The infinite system is truncated to a finite one. The method does not apply any auxiliary constructions or specific conformal mappings, it does not use the accessory solutions of boundary value problems and it does not require iterations. It allows us to construct and compute the conformal mappings onto different multi-connected domains without recursion and other iterative procedures. The accuracy similar to that of [23] can be achieved with a matrix of the size of 200. The resulting approximate solution presented here is an analytic function with all of its properties such as possessing derivatives of any order. The natural replacement of all the features in our case is a solution of a large linear system.

\section{References}

[1] Schinzinger, R., \& Laura, P.A.A. (2003). Conformal Mapping: Methods and Applications. Dover Publications.

[2] Mityushev, V. (1998). Convergence of the Poincare series for classical Schottky groups. Proc. AMS , 126, 2399-2406. 
[3] Mityushev, V., \& Rogosin, S., (1999). Constructive Methods for Linear and Nonlinear Boundary-Value Problems for Analytic Functions. Theory and Applications. Chapman \& Hall/CRC.

[4] Mityushev, V. (2014), Poincare $\alpha^{*}$-series for classical Schottky groups and its applications, In Milovanović, G.V., Rassias M.Th. (Eds.). Analytic Number Theory, Approximation Theory, and Special Functions. Springer.

[5] Goluzin, G.M. (1969). Geometric Theory of Functions of a Complex Variable. AMS.

[6] Murid, A.H.M., \& Laey-Nee Hu (2009). Numerical conformal mapping of bounded multiply connected regions by an integral equation method. Int. J. Contemp. Math. Sci., 4, 1121-1147.

[7] Henrici, P. (1986). Applied and Computational Complex Analysis, Vol. 3. New York: Wiley.

[8] Wegmann, R. (2001) Fast conformal mapping of multiply connected regions. J. Comput. Appl. Math., 130, 119-138.

[9] Wegman, R., \& Nasser M.M.S. (2008). The Riemann-Hilbert problem and the generalized Neumann kernel on multiply connected regions. J. Comput. Appl. Math., 214, 36-57.

[10] DeLillo, T.K. (1994). The accuracy of numerical conformal mapping methods: A survey of examples and results. SIAM J. Num. Anal., 31 (3), 788-812.

[11] DeLillo, T.K. (1987). On some relations among numerical conformal mapping methods. J. Comput. Appl. Math., 19, 363-377.

[12] Yunus, A.A.M., Murid, A.H.M., \& Nasser, M.M.S. (2014). Numerical conformal mapping and its inverse of unbounded multiply connected regions onto logarithmic spiral slit regions and rectilinear slit regions. Proc. of the Royal Society A - Math. Phys. and Eng. Sci., 470(2162), Article No. 20130514.

[13] Shirokova, E.A., \& Ivanshin, P.N. (2016). Approximate conformal mappings and elasticity theory. J. Compl. Analysis, ID 4367205.

[14] Abzalilov, D.F., \& Shirokova, E.A. (2017). The approximate conformal mapping onto simply and doubly connected domains. Complex Variables and Elliptic Equations, 62, 554-565.

[15] Gakhov, F.D. (1966). Boundary Value Problems. Oxford: Pergamon Press.

[16] Sangawi, A.W.K., Murid, A.H.M., \& Nasser, M.M.S. (2012). Annulus with circular slit map of bounded multiply connected regions via integral equation method. Bull. Malays. Math. Sci. Soc., 35, 945-959.

[17] Yunus, A.A.M., Murid, A.H.M., \& Nasser, M.M.S. (2014). Numerical evaluation of conformal mapping and its inverse for unbounded multiply connected regions. Bull. Malays. Math. Sci. Soc., 1(24), 1-24.

[18] Wegmann, R. (2005). Methods for Numerical Conformal Mappings. Handbook of Complex Analysis: Geometric Function Theory, 2, Amsterdam, The Netherlands: Elsevier, 351-477.

[19] Abzalilov, D., \& Shirokova, E. (2019). The approximate conformal mapping onto multiply connected domains. Prob. Anal. Issues Anal., 8(1), 3-16.

[20] von Koppenfels, W., \& Stallmann, F. (1959). Praxis der konformen Abbildung. Berlin: Springer-Verlag.

[21] Shirokova, E.A. (2014). On the approximate conformal mapping of the unit disk on a simply connected domain. Russian Math., 58(3), 47-56.

[22] El-Shenawy, A., \& Shirokova, E.A. (2018). A Cauchy integral method to solve the 2D Dirichlet and Neumann problems for irregular simply-connected domains. Uchenye Zapiski Kazanskogo Universiteta. Ser.Fiz-Mat.N., 160(4), 778-787.

[23] Nasser, M.M.S. (2011). Numerical conformal mapping of multiply connected regions onto the second, third and fourth categories of Koebe's canonical slit domains. J. Math. Anal. Appl., 382, 47-56. 